\title{
Mutation analysis and characterization of $H S D 17 B 2$ sequence variants in breast cancer cases from French Canadian families with high risk of breast and ovarian cancer
}

\author{
Marie Plourde ${ }^{1}$, Caroline Manhes ${ }^{1}$, Gilles Leblanc ${ }^{1}$, Francine Durocher ${ }^{1}$, Martine Dumont ${ }^{1}$, \\ Olga Sinilnikova ${ }^{2}$, INHERIT BRCAs ${ }^{*}$ and Jacques Simard ${ }^{1,3}$ \\ ${ }^{1}$ Cancer Genomics Laboratory, Oncology and Molecular Endocrinology Research Center, Centre Hospitalier Universitaire de Québec and Laval University, Quebec G1V 4G2, Canada \\ ${ }^{2}$ Plate-Forme Mixte de Génétique Constitutionnelle des Cancers Fréquents, Hospices Civil de Lyon/Centre Léon Bérard, 69373 Lyon Cedex 08 , France \\ ${ }^{3}$ Canada Research Chair in Oncogenetics \\ (Correspondence should be addressed to J Simard who is now at Cancer Genomics Laboratory, CHUL Research Center, CHUQ, 2705 Laurier Boulevard, T3-57, Quebec City, Quebec \\ G1V 4G2, Canada; Email: jacques.simard@ crchul.ulaval.ca) \\ *Other members of INHERIT BRCAs involved in clinical aspects of the program are listed in the Appendix
}

\begin{abstract}
Estrogen exposure is a risk factor for breast cancer. Given that HSD17B2 gene encodes an enzyme that catalyses estradiol inactivation, it appears as a good candidate breast cancer susceptibility gene. This study was designed to screen for HSD17B2 germline mutations potentially involved in breast cancer predisposition. Our re-sequencing analysis did not identify any deleterious germline mutations, and therefore mutations in HSD17B2 do not explain the clustering of breast cancer cases in non-BRCA1/2 high-risk French Canadian families. However, six sequence variants were identified, including two novel missense variants. Expression assays revealed that p.Ala111Asp and p.Gly 160 Arg did not alter the catalytic properties of $17 \beta$-hydroxysteroid dehydrogenase type 2 enzyme, although p.Ala111Asp appears to affect protein stability resulting in significant decreases in the protein levels, providing valuable information on structurefunction relationship.
\end{abstract}

Journal of Molecular Endocrinology (2008) 40, 161-172

\section{Introduction}

Most of the familial aggregation in breast cancer results predominantly from inherited susceptibility (Lichtenstein et al. 2000, Peto \& Mack 2000). Germline mutations in high-penetrance cancer susceptibility genes such as breast cancer 1 gene (BRCA1), breast cancer 2 gene (BRCA2), tumor protein p53 (TP53), phosphatase and tensin homolog $(P T E N)$, and ataxia telangiectasia mutated gene $(A T M)$ are associated with some of the familial breast cancer cases, although this association accounts for $\sim 25 \%$ of the familial component of breast cancer risk (Pharoah et al. 2004, Thompson \& Easton 2004, Antoniou \& Easton 2006).

It has been proposed that a complex polygenic model may be the best explanation for this missing genetic risk (Antoniou et al. 2001). Under the common variant/ common disease $(\mathrm{CV} / \mathrm{CD})$ model, disease susceptibility is suggested to result from the joint action of several CVs, with unrelated affected individuals sharing a substantial proportion of disease alleles (Cargill \& Daley 2000, Reich \& Lander 2001, Lohmueller et al. 2003, Easton et al. 2007). The alternative is the heterogeneity hypothesis, which maintains that genetic susceptibility to $\mathrm{CD}$ is caused by many different rare genetic variants with a relatively large effect produced by each allele (Pritchard 2001, Pritchard \& Cox 2002, Fearnhead et al. 2004). This hypothesis will be more easily detectable by the candidate gene re-sequencing approach (Renwick et al. 2006, Seal et al. 2006, Rahman et al. 2007).

Both epidemiological and cell biology experiments have documented the contribution of estrogens in the regulation of cellular growth, differentiation and proliferation of the normal mammary gland as well as in hormone-sensitive breast carcinomas, while androgens rather exert an anti-proliferative action in these tissues (Doisneau-Sixou et al. 2003, Labrie et al. 2003). There is a large body of evidence showing that in humans, the local intracrine formation of active estrogens and androgens from inactive steroid precursors secreted by the adrenals, namely, dehydroepiandrosterone and its sulfate, regulates the growth and function of peripheral target tissues, including the breast (Labrie et al. 2003).

Among the enzymes modulating local sex steroid availability, $17 \beta$-hydroxysteroid dehydrogenase type 2

DOI: 10.1677/JME-07-0101 Online version via http://www.endocrinology-journals.org 
enzyme (17 $\beta$-HSD) that is responsible for the inactivation of estradiol (the more potent estrogen) into estrone, is located on chromosome 16q23.3-q24.1, a genomic region showing frequent loss of heterozygosity in breast cancer (Suzuki et al. 1996, Sato et al. 1998, Cleton-Jansen et al. 2001). For oxidative reactions in intact cells, 17 $\beta$-HSD type 2 prevails in its kinetic parameters over other forms of 17ß-HSDs (Wu et al. 1993, Labrie et al. 1995, Mindnich et al. 2004). HSD17B2 is expressed both in normal breast tissue and in malignant breast tumors and it has been suggested that it may protect normal breast cells from the effects of excessive estradiol production (Casey et al. 1994, Miettinen et al. 1999, Gunnarsson et al. 2001, Oduwole et al. 2004). It should be noted that tumors frequently lack detectable levels of $17 \beta-H S D$ type 2 and in cases where its expression is retained, there is evidence of a decreased risk for late relapse of breast cancer (Gunnarsson et al. 2001). Among estrogen receptor-positive patients, those with a low expression of $17 \beta$-HSD type 2 had a significantly higher recurrence rate when compared with patients who expressed normal levels (Gunnarsson et al. 2005). An increase or decrease in this enzyme activity may therefore alter the levels of endogenous estrogens, thereby influencing breast cancer susceptibility.

Studies investigating the role of genetic variants in the $H S D 17 B 1$ gene, which gives rise to $17 \beta-H S D$ type 1 enzyme that predominantly catalyses the reduction of estrone to estradiol, have been conducted in relation to breast cancer risk with non-conclusive results (Mannermaa et al. 1994, Feigelson et al. 2001, 2006, Wu et al. 2003, Setiawan et al. 2004, Plourde et al. 2007). On the other hand, when this study began, there were no studies investigating the presence of HSD17B2 germline mutations or polymorphisms in families with high risk of breast cancer. Based on the pivotal role of HSD17B2 in modulating local sex steroid availability in the breast tissue, $H S D 17 B 2$ represents an attractive candidate gene to potentially explain a fraction of the remaining familial component of breast cancer risk. We therefore performed comprehensive re-sequencing of the HSD17B2 gene in women affected with breast cancer from nonrelated BRCA1- and BRCA2-negative French Canadian families with a high risk of breast or ovarian cancer.

\section{Materials and methods}

\section{Ascertainment of high-risk families and DNA extraction}

The recruitment of French Canadian families with a high risk of breast and ovarian cancer started in 1996 through a research project, which thereafter evolved in a large ongoing interdisciplinary research program designated INHERIT BRCAs. More details regarding ascertainment criteria, experimental and clinical procedures, as well as the INHERIT BRCAs research program have been described elsewhere (Simard et al. 2007). A major component was to identify and characterize the prevalence and penetrance of BRCA1 and BRCA2 mutations in French Canadian high-risk families (Antoniou et al. 2006, Simard et al. 2007). Subsequently, another component was designed for the 'localization and identification of new breast cancer susceptibility loci/genes'. Ethics approval was obtained from the different institutions participating in this research component and each participant had to sign an informed consent to participate in this latter project (Durocher et al. 2006). A subset of 50 high-risk French Canadian breast/ovarian cancer families were recruited in the present study according to the presence of multiple cases of breast cancer, among which 45 included at least 3 individuals with breast cancers within second degree relatives, while 5 families included 3 or more individuals within third degree relatives. All participants had to be at least 18 years of age and mentally capable. The diagnoses of breast and/or ovarian cancer were confirmed by a pathology report, and when two or more subjects were available within a family, the youngest subject was systematically chosen for this study. The mean age at diagnosis of these 50 subjects affected with breast cancer was $47 \cdot 7$ years (34-69 years), while 35 of them have been diagnosed before 50 years of age and 8 had bilateral breast cancer.

The BRCA1/2 status of each participant was assessed previously (Simard et al. 2007). The evidence of the absence of genomic rearrangements in $B R C A 1 / 2$ genes was thereafter investigated by multiplex ligation-dependent probe amplification (MLPA) for 44 out of the 50 subjects (BRCA1/2 Southern analyses were done for 32 out of these 50 subjects). For four of the remaining subjects, MLPA was performed on another individual of the family (Moisan et al. 2006), while for two subjects this analysis was not performed in their family.

Genomic DNA from 73 healthy unrelated French Canadian individuals was obtained from Dr Damian Labuda at the Centre de Cancérologie Charles Bruneau, Hôpital Ste-Justine, Montreal, Canada. The individuals who provided these samples were recruited on a nonnominative basis, in the framework of long-term studies aiming the characterization of the genetic variability in human populations, approved by the Institutional Ethic Review Board. The mean age of these individuals was 43.3 years; $4(5.5 \%), 24(32.9 \%), 27(37.0 \%), 13(17.8 \%)$, and $5(6 \cdot 8 \%)$ of them were between 25-29, 30-39, 40-49, $50-59$, and $60-69$ years of age respectively.

\section{PCR amplification, mutation analysis, and sequence variant identification}

The complete sequence of the five coding exons, flanking intronic sequences, $5^{\prime}$ and $3^{\prime}$ non-coding regions and $1000 \mathrm{bp}$ of the promoter region of the HSD17B2 gene was 
analyzed in DNA samples obtained from Epstein-Barr virus (EBV)-transformed B-lymphoblastoid cell lines of 50 affected individuals. Five primer pairs were used to amplify the fragments that were sequenced in both directions with primers indicated in Table 1. The frequency of variants was also ascertained in 73 healthy unrelated French Canadians. After the completion of this study, Jansson et al. (2007) published their results from the HSD17B2 mutation screening among breast cancer cases from Sweden, which reported a previously unknown missense variant (p.Met226Val) not observed among our affected individuals. The presence of this variant was subsequently ascertained in 69 out of our 73 healthy controls. HSD17B2 direct sequencing was performed on an ABI Prism 3730 DNA Analyser automated sequencer using version 3.1 of the Big Dye fluorescent method according to the manufacturer's instructions (Applied Biosystems, Foster City, CA, USA). Sequence data were analyzed using the Staden preGap4 and Gap4 programs. The GenBank accession number for the HSD17B2 reference sequences used in these experiments was NT_010498.15 and NM_002153.1 respectively.

The region of the HSD17B2 gene including the polymorphic CA repeat (single nucleotide polymorphism, SNP 1) was amplified using the following primers: forward, 5'-GTAGGAGTCCTGAAGCTATGG-3' and reverse, 5'-GTTTCTTGAACTGCATGGAGAATAACAG-3' of which the forward primer was labeled with Applied Biosystems fluorescent dye (5-carboxy-fluorescein; FAM). Typically, $20 \mathrm{ng}$ genomic DNA was used in each PCR of $15 \mu \mathrm{l}$. The PCR protocol involved a denaturation step at $95^{\circ} \mathrm{C}$ for $5 \mathrm{~min}$ and then 35 cycles of $95^{\circ} \mathrm{C}$ for $30 \mathrm{~s}$ followed by $40 \mathrm{~s}$ at $57^{\circ} \mathrm{C}$, with a final elongation cycle of $72^{\circ} \mathrm{C}$ for $8 \mathrm{~min}$. Each reaction contained $2.3 \mathrm{mM} \mathrm{MgCl}{ }_{2}, 1 \mathrm{U}$ platinum Taq polymerase (Invitrogen), $1 \times \mathrm{PCR}$ buffer without $\mathrm{MgCl}_{2}, 0 \cdot 1 \mathrm{mM}$ dNTP and 2 pmol of each primer. PCR products were diluted to obtain a 1: 250 ratio. From this dilution, $2 \mu \mathrm{l}$ were added to $10 \mu \mathrm{l}$ Applied Biosystems HiDi-formamide containing Genescan 500 LIZ (3:1000 dilution). Samples were resolved on an ABI Prism 3100 Genetic Analyzer and data were analyzed using the GeneMapper Version 2 software (Applied Biosystems). Several samples were sequenced to confirm the repeat length as established by the ABI 3100 Genotyper.

\section{LD analysis, haplotype estimation, and in silico analysis tools}

To estimate the pattern of linkage disequilibrium (LD), we used the LDA program (http://www.chgb. org.cn/lda/lda.htm; Ding et al. 2003), which calculates pairwise LD for the common sequence variant pairs. Lewontin's standardized disequilibrium coefficient $\left|D^{\prime}\right|$ (Lewontin 1964, Devlin \& Risch 1995) and the square of correlation coefficient $r^{2}$ (Hill \& Robertson 1968) were used as measures of LD between variants. Haplotype reconstructions and frequency estimations were performed using the PHASE 2.1.1 software (http://www.stat.washington.edu/stephens/; Stephens et al. 2001). This program (PHASE) estimates haplotype frequencies with a Bayesian-based algorithm and then uses a permutation test to determine the significance of differences in the inferred haplotypes between cases and controls. Computations were made using the software's default parameters.

Splice site prediction scores were evaluated using splice site prediction by neural network (SSPNN; http://www. fruitfly.org/seq_tools/splice.html) and Alex Dong Li's splice site finder (http://www.genet.sickkids.on.ca/ali/ splicesitefinder.html; Shapiro \& Senapathy 1987), while the effect of amino acid substitutions was evaluated using sorting intolerant from tolerant (SIFT; http://blocks. fhcrc.org/sift/SIFT.html; Ng \& Henikoff 2001) and PolyPhen (http://genetics.bwh.harvard.edu/pph/; Ramensky et al. 2002).

\section{Site-directed mutagenesis}

The following oligonucleotide sequences were designed such that the desired mutation was in the

Table 1 Oligonucleotide primers used for amplification and sequence analysis of the HSD17B2 gene

Forward primer $\left(5^{\prime}-3^{\prime}\right)$

\section{Amplified region ${ }^{a}$} Promoter and exon 1

Exon 2

Exon 3

Exon 6

Exon 7
Reverse primer $\left(5^{\prime}-3^{\prime}\right)$

CAAGTTTCAGATCATACCCTCAGT $^{\mathrm{b}}$
GCACCTCTGATCTGGTCCATAG $^{\mathrm{G}}$
GCAGCCTGAGTTCTGTGAACAG $^{\mathrm{c}}$
GTTACTCCGAAGGTCTTCATG $^{\mathrm{b}}$
AACCCTGCCTTTGCATGCCCTG $^{\mathrm{b}}$
TGAGGGGTTCAATGTGTGCTCTG $^{\mathrm{b}}$
CCTCAATTCTCTGATGAGAACTAG $^{\mathrm{b}}$

Product length (bp)

GCATTTATGAGGTTCCTTCTACTG AGAATGCTTAGGGGCATACAG ${ }^{\circ}$ AGAGCGGTCAAATAGGAAGTGCCTTG ${ }^{\circ}$ GCATGGAGAATCGTAAACAACG ${ }^{\mathrm{b}}$ TGCATGGTCTTCCAGGTATTG ${ }^{\mathrm{b}}$ AGACACTGATTAAATATTTATTGG ${ }^{b}$ AGGGCCATCCTTCCCAACAGAGACAAG ${ }^{b}$
1846

491

371

307

699

aAnnealing temperature at $60^{\circ} \mathrm{C}$

bUsed as amplification and sequencing primers.

${ }^{c}$ Used as sequencing primer only. 
middle of the primer with about $10-15$ bases of specific sequence on either side. The primer sequences were as follows. For the $\mathrm{C}$ to A mutation of c.332C $>\mathrm{A}$ : forward (5'-GCTTCACGGTATTTGACGGAGTTTTGAATG-3') and reverse (5'-CATTCAAAACTCCGTCAAATACCGTGAAGC-3' ${ }^{\prime}$; for the $\mathrm{G}$ to $\mathrm{C}$ mutation of $\mathrm{c} .478 \mathrm{G}>\mathrm{C}$ : forward (5'-GCAGGACAGACGACTGTGGGC-3') and reverse (5'-GCCCACAGTCGTCTGTCCTGC-3'). Sitedirected mutagenesis was performed on an expression vector containing the full-length cDNA fragment encoding the complete amino acid sequence for human $17 \beta$-HSD type 2 (kindly provided by Dr Van Luu-The, CHUL Research Center, Quebec, Canada) inserted in the pcDNA3.1 vector (Invitrogen) using the QuickChange Site-directed mutagenesis kit from Stratagene (Stratagene Cloning Systems, La Jolla, CA, USA) according to the supplier's protocol. Insertion of the mutation was confirmed by direct sequencing using Big Dye Terminator chemistry on an ABI Prism 3730 automated sequencer from Applied Biosystems.

\section{Transcription/translation}

Transcription/translation was performed using the TNT Quick coupled transcription/translation system from Promega according to the manufacturer's instructions, as described previously (Plourde et al. 2007).

\section{Cell culture and transfection}

Human embryonic kidney 293 cells (HEK293) were obtained from the American Type Culture Collection (ATCC; Manassas, VA, USA) and were cultured in Dulbecco's modified Eagle's medium (DMEM)/high glucose from Invitrogen Life Technologies Inc. supplemented with $10 \%$ fetal bovine serum (FBS), $1 \%$ glutamine, $100 \mathrm{IU} / \mathrm{ml}$ penicillin, and $50 \mathrm{mg} / \mathrm{ml}$ streptomycin from WISENT Inc. (St-Bruno, Quebec, Canada) until confluence. The cells were passaged and plated in $175 \mathrm{~cm}^{2}$ culture dishes at a density of $9 \cdot 1 \times 10^{6}$ cells. The cells were allowed to settle overnight after which the medium was changed to DMEM without FBS just before transfection. Transient transfection was performed using ExGen 500 cationic polymer transfection reagent (MBI Fermentas Inc., Ontario, Canada) according to the supplier's protocol. The cells were also transfected with the $\mathrm{pcDNA}_{3}$ vector as a negative control. One day after transfection, the cells were scrapped to assess enzyme activity, as detailed below.

\section{Western analysis}

Western analysis of proteins was performed by SDSPAGE on discontinuous acrylamide gels, as described previously (Plourde et al. 2007). Briefly, total proteins were extracted from HEK293 cells in PBS and cell lysis was performed using standard procedures. Total proteins $(15 \mu \mathrm{g})$ were separated on a $4 \%$ stacking and $12 \%$ resolving gel and transferred onto a nitrocellulose membrane (Hybond-ECL, Amersham Pharmacia Biotech Inc.) and thereafter hybridized to a polyclonal antibody directed against human 17ß-HSD type 2 (kindly provided by Dr Van Luu-The) at a dilution of 1:1000 and subsequently incubated with a donkey antirabbit immuno-globulin G (IgG) peroxidase-conjugated secondary antibody (Amersham Pharmacia Biotech Inc.) at a dilution of 1:10 000. Membranes were washed and proteins were visualized using the SuperSignal West Dura Extended Duration Substrate kit (PIERCE, Rockford, IU, USA), followed by exposure of the membranes to X-ray films for 1-10 min. The autoradiographic film was scanned and the Image J program (NIH, Bethesda, MD, USA) was used to quantify the density of the autoradiographic bands.

\section{Assay of $17 \beta-$ HSD type 2 enzymatic activity}

In order to measure $17 \beta$-HSD type 2 activity, the amount of translated mutant recombinant proteins have been adjusted to be equivalent to $10 \mu \mathrm{g}$ total protein of normal 17 $\beta$-HSD type 2 enzyme in each corresponding cell homogenate preparation, as measured previously by immunoblot analysis. The cell homogenates were incubated for $0.5-4 \mathrm{~h}$ at $37^{\circ} \mathrm{C}$ in the presence of $10 \mathrm{nM}\left[4^{14} \mathrm{C}\right]$-estradiol $(52 \cdot 0 \mathrm{mCi} / \mathrm{mmol})$ from Perkin-Elmer Life Sciences Inc. (Boston, MA, USA), $1 \mathrm{mM} \mathrm{NADH}\left(\mathrm{NAD}^{+}\right)$cofactor, and a buffer

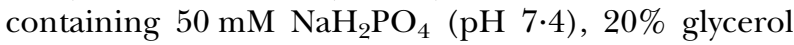
( $\mathrm{vol} / \mathrm{vol})$ and $1 \mathrm{mM}$ EDTA. After the indicated time intervals, steroids were extracted by the addition of 2 volumes of diethyl ether and the incubation mixture was chilled in a dry ice/ethanol bath. Steroids were separated by thin layer chromatography using a mobile phase of toluene:acetone $(4: 1)$ and analyzed using phosphoimaging, Storm 860 (Molecular Dynamics Inc., Sunnyvale, CA, USA). All results are expressed as the mean \pm s.E.M. of at least three separate transfection experiments performed in triplicate.

\section{Results}

\section{HSD17B2 mutation analysis and variant characterization}

Although no truncating mutation was found in the HSD17B2 coding region of our French Canadian breast cancer cases, we identified six variants in $H S D 17 B 2$ exonic, flanking intronic, and promoter sequences (Fig. 1A). 
A
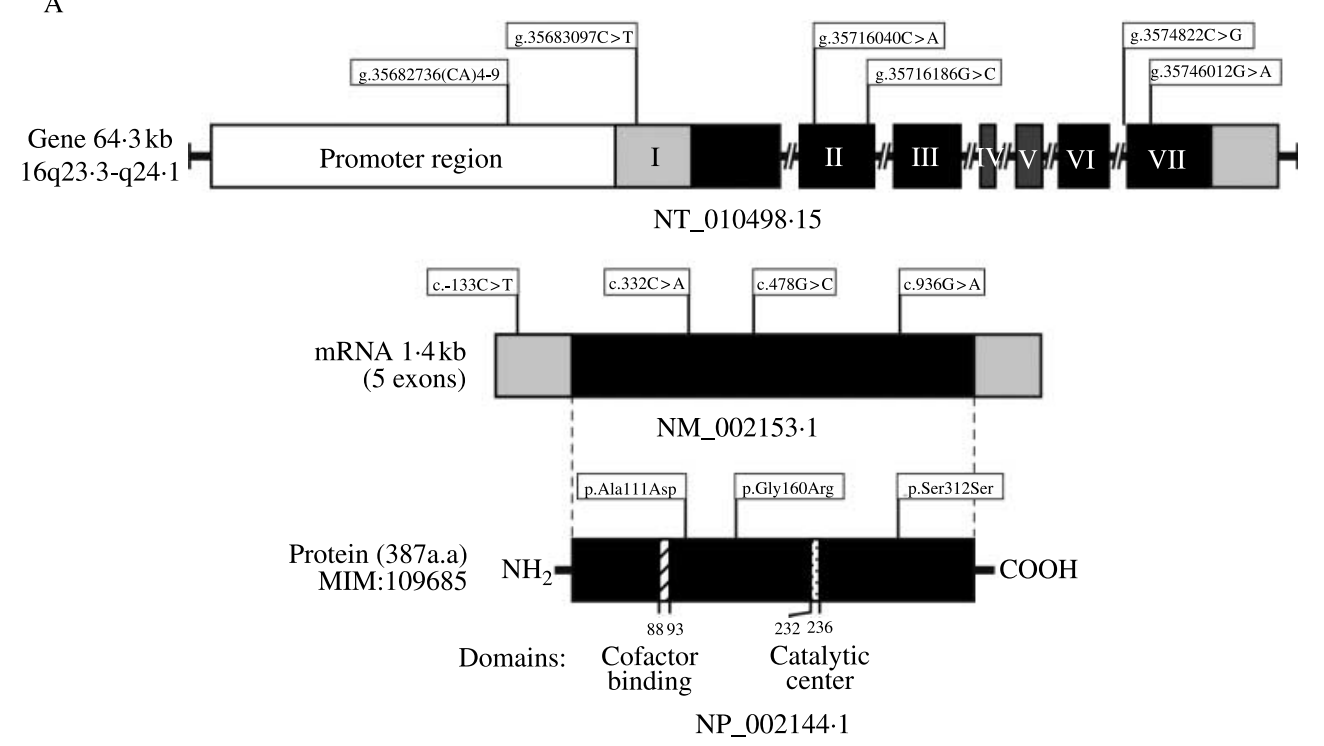

B

\begin{tabular}{ccc}
\hline & p.Ala111Asp & p.Gly160Arg \\
\cline { 2 - 3 } Pan troglodytes & VFAGV & DRGLW \\
Canis familiaris & VFVGV & DRGLW \\
Rattus norvegicus & VFAGV & DKGLW \\
Mus musculus & VFAGV & DKGLW \\
Bos taurus & VFAGV & NKGLW \\
Monodelphis domestica & VFVGV & QTGIR \\
Gallus gallus & VFAGV & KTGLW \\
Xenopus tropicalis & VFAGV & DAGLW
\end{tabular}

Figure 1 (A) Genomic structure of the human HSD17B2 gene. The promoter is indicated by a white box and introns by solid broken lines. Exons are represented by proportionally sized boxes. Exons 1-3 and 6-7, encoding active $17 \beta$-HSD type 2 , are represented by dark boxes, whereas the two additional exons (4-5) from the non-functional transcript are illustrated in dark gray. The $5^{\prime}$ and $3^{\prime}$ untranslated regions are in light gray in the gene and mRNA structure respectively. The GenBank accession numbers corresponding to the HSD17B2 gene contig, the mRNA and the protein are indicated below the nucleotide and protein sequences. The domains involved in the $17 \beta-H S D$ type 2 protein activities are illustrated on the protein structure. All sequence variants are indicated in open boxes while amino acid nomenclature is also represented. (B) Non-synonymous sequence variants detected in human $17 \beta-H S D$ type 2 and residues found in orthologs.

These included a CA repeat located in the promoter region (arbitrarily defined as a 1000 bp genomic segment upstream exon 1), a common nucleotide substitution in the $5^{\prime}$-untranslated region $(\mathrm{c} .-133 \mathrm{C}>\mathrm{T})$, two rare non-synonymous variants in exon 2 (c.332C $>$ A (p.Ala111Asp) and c.478G $>$ C (p.Gly160Arg)), one common substitution in intron $6(\mathrm{c} .803-57 \mathrm{C}>\mathrm{G})$ and one rare synonymous sequence variant located in exon 7 (c.936G > A; Table 2). Two were novel, while four were reported in the SNP databases (dbSNP; www.ncbi.nlm.nih.gov/SNP). Genotype and minor allele frequencies (MAF) were determined in cases and controls, both of French Canadian origin. Frequencies were similar with frequencies reported in dbSNP database. As indicated in Table 2, the distribution of all genotypes was in accordance with Hardy-Weinberg equilibrium.

\section{Effect of missense substitutions p.Ala111Asp and p.Gly160Arg on $17 \beta-H S D$ activity and expression levels}

Two non-synonymous SNPs were observed in the coding sequence of HSD17B2 (SNP \#3 and SNP \#4), and both were only observed once at a heterozygous state (Table 2). These two cSNPs resulted in changes in encoded amino acids, p.Ala111Asp and p.Gly160Arg. Segregation of the missense variants with breast cancer was analyzed in 
Table 2 Observed sequence variants and genotype frequencies in HSD17B2 gene among familial breast cancer cases and controls

\section{Genotype frequencies}

SNP ID

dbSNP ID

zygotes $(\operatorname{Exp})^{\mathrm{c}}$

Heterozygotes
$(\text { Exp })^{c}$

Rare homo-

zygotes $(\operatorname{Exp})^{c}$

MAF

$\chi^{2} P$

value $^{d}$

Reported

MAF $^{\mathrm{e}}$

SNP

g.35682736(CA)4-9

rs10595118 Promoter

Cases

Controls

$0.37(0.37)$

$0.37(0.37)$
$0.39(0.36)$

$0.49(0.48)$

$0.43(0.48)$

$0 \cdot 14(0 \cdot 15)$

$0.18(0.16)$

0.39
0.40

0.83

$0.36(0.37)$

$0.50(0.48)$

$0.14(0.15)$

$0.12(0 \cdot 13)$

0.39
0.36

0.40

Controls $\quad 0.40(0.41)$

$0.48(0.46)$

$0.00(0.00)$

$0.00(0.00)$

$0 \cdot 01$

$0.02(0.02)$
$0.00(0.00)$

Controls $\quad 1.00(1.00)$

N/A

Exon 2

Cases

$0.98(0.98)$

$0.02(0.02)$

Controls

$1.00(1.00)$

$0.00(0.00)$

$0.00(0.00)$

$0.00(0.00)$

0.00

rs8191243 Intron 6

g.35745822C $>G$

c. $803-57 \mathrm{C}>\mathrm{G}$

6 g. $35746012 \mathrm{G}>\mathrm{A}$

c. $936 \mathrm{G}>\mathrm{A}$
Cases

Controls

$0.40(0.44)$
$0.48(0.50)$

Cases

Controls
$0.98(0.98)$

$0.99(0.98)$
$0.52(0.45)$

$46(0.41)$

$0.02(0.02)$

$0.01(0.02)$

$0.08(0.12)$

$0.06(0.08)$

$0.00(0.00)$

$0.00(0.00)$

$\mathrm{N} / \mathrm{A}$, information not available for this SNP (not reported in dbSNP). MAF, minor allele frequency.

${ }^{a}$ According to the nomenclature of the Human Genome Variation Society, for cDNA numbering +1 corresponds to the A of the ATG translation initiation codon in the reference sequence. ${ }^{b}$ Observed among 100 chromosomes from 50 breast cancer cases and 146 chromosomes from 73 women controls DNA samples.

${ }^{\mathrm{c}}$ As expected under Hardy-Weinberg equilibrium.

${ }^{\mathrm{d}} P$ value for deviation from Hardy-Weinberg equilibrium (Pearson's $\chi^{2}$ ).

${ }^{e}$ Minor allele frequencies are reported from www.hapmap.org using the $\mathrm{CEPH}$ population and www.ensembl.org with the Perlegen European panel.

${ }^{\mathrm{f}}(\mathrm{CA})_{9}$, common allele, $(\mathrm{CA})_{8}$, rare allele; $(\mathrm{CA})_{7}$ was observed once in controls while $(\mathrm{CA})_{4}$ was observed twice in cases, these data are not included in calculated frequencies. 
respective families in order to determine their potential involvement in the modification of breast cancer risk (data not shown). In the p.Ala111Asp variant carrier's family, the variant does not segregate with breast cancer, in fact, the participant's sister was also diagnosed with breast cancer at 42 years of age and does not carry the mutation. The same observation applies to the p.Gly160Arg variant carrier's family in which the participant's sister has also developed breast cancer at an early age (38 years), while she is a common homozygote Gly/ Gly. These observations thus do not support the implication of these sequence variants in the clustering of breast cancer in these high-risk families.

In order to obtain more information on structurefunction relationships, we performed several analyses for these missense variants. The two amino acid substitutions are located outside the known sequence motifs of the short chain dehydrogenase/reductase family of which $17 \beta$-HSD type 2 protein is a member (Fig. 1A; Oppermann et al. 2001). However, in order to obtain a more representative prediction of the importance of specific residues on protein function, comparison of missense substitutions was performed across relevant species. Alignment of 17ß-HSD type 2 ortholog sequences revealed that p.Ala111 and p.Gly160 are conserved amino acids in vertebrates thus suggesting that these positions are under strong functional constraint (Fig. 1B). Furthermore, for the p.Gly160Arg variant, unlike arginine, glycine is not a charged residue and has a very different structure, while for the p.Ala111Asp variant, alanine and asparagine are both hydrophobic and charged amino acids. In silico analysis revealed that for both variants, the change in amino acid is predicted to be damaging to protein structure $(\mathrm{Ng} \&$ Henikoff 2001, Ramensky et al. 2002). Moreover, p.Gly160Arg (c.478G $>$ C) is located at the boundary of an exon-intron splice recognition sequence. We evaluated in silico the potential consequence of this variant on splicing by calculating the splicing efficiencies in the normal and mutant sequences with two splice prediction programs: the SSPNN from the Berkeley Drosophila Genome Project and Alex Dong Li's Splice Site Finder (Shapiro \& Senapathy 1987). Both programs predicted that this SNP abolished the normal donor splicing site of exon 2, suggesting a loss of the splicing-site recognition property. In order to further investigate whether the p.Gly160Arg (p.478G $>$ C) variant may lead to alternative splicing on mRNA, we performed a PCR on cDNA from immortalized cell lines from the heterozygous individual as well as from wild-type individuals, in the region surrounding the p.Gly160Arg (c.478G $>$ C) variant. No transcript of wild-type $H S D 17 B 2$ could be detected in immortalized cell lines and therefore the effect of the p.Gly160Arg (c.478G $>$ C) variant on splicing could not be confirmed. Note that such in silico analysis was also carried out to investigate the potential effect of SNP \#5 (c.803-57C > G) located in intron 6, on splicing. This analysis predicted that this variant had no effect on splicing-site recognition properties.

Functional assays were performed to investigate the effect of the amino acid substitutions resulting from the two non-synonymous variants on 17 $\beta$-HSD type 2 recombinant products. In vitro transcription/translation assays show normal expression levels of both wildtype and mutant recombinant p.Ala111Asp and p.Gly160Arg proteins (Fig. 2A). A $43 \mathrm{kDa}$ band corresponding to $17 \beta$-HSD type 2 protein was detected by western blot analysis using cells expressing the wildtype and p.Gly160Arg variant, whereas a very weak signal was detectable for the mutant recombinant protein p.Ala111Asp (Fig. 2B). Similar expression levels were observed in at least five independent experiments, as well as in the presence of proteasome and protease inhibitors (data not shown). We also investigated the time course formation of $\left[{ }^{14} \mathrm{C}\right]$-estrone from $\left[{ }^{14} \mathrm{C}\right]-$ estradiol in transfected homogenated HEK293 cells. As illustrated in Fig. 2C, the apparent activities of p.Ala111Asp and p.Gly160Arg enzymes were similar to that of the wild-type enzyme. These results for p.Ala111Asp indicate that this substitution may probably yield a much less stable enzyme that could be rapidly degraded, but that otherwise does not affect its enzymatic capacities when the amount of translated mutant recombinant protein is corrected to be equivalent to the wild-type.

\section{LD and haplotype analyses}

Tagging SNP analysis is a way of maximizing the study of genetic variability irrespective of the functionality of individual SNPs. Indeed, haplotype prediction and tSNP selection using common SNPs (MAF $\geq 5 \%$ ) provides good power to capture common variations in a gene and therefore allows the use of these tSNPs in studies involving other populations. Furthermore, since all genotyped SNPs are in strong LD with one of the tSNPs, although the causal variant may not be genotyped, it will be indirectly represented by one of the tSNPs. The estimation of haplotypes may also allow the observation of a phenotype associated with a combination of SNPs rather than with a single SNP.

In order to do so, we must first determine $\mathrm{LD}$ values between SNPs and infer major haplotypes, to determine SNPs that will tag these haplotypes. Pairwise LD values involving the two CVs, SNPs 2 and 5 (excluding the CA repeat), were calculated, while $\mathrm{LD}$ values for pairs with variants 3, 4, and 6 were not since each one was observed in only a single heterozygous case. When measured by Lewontin's $\left|D^{\prime}\right|$, these sequence variants were in strong $\mathrm{LD}$ with each other $\left(D^{\prime}=0.61\right.$ for breast cancer cases and $D^{\prime}=1 \cdot 00$ for controls) even with an inter-marker distance of $\sim 63 \mathrm{~kb}$, suggesting that $\mathrm{LD}$ 
A

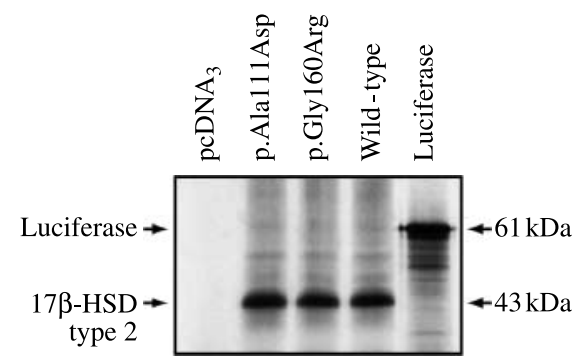

B
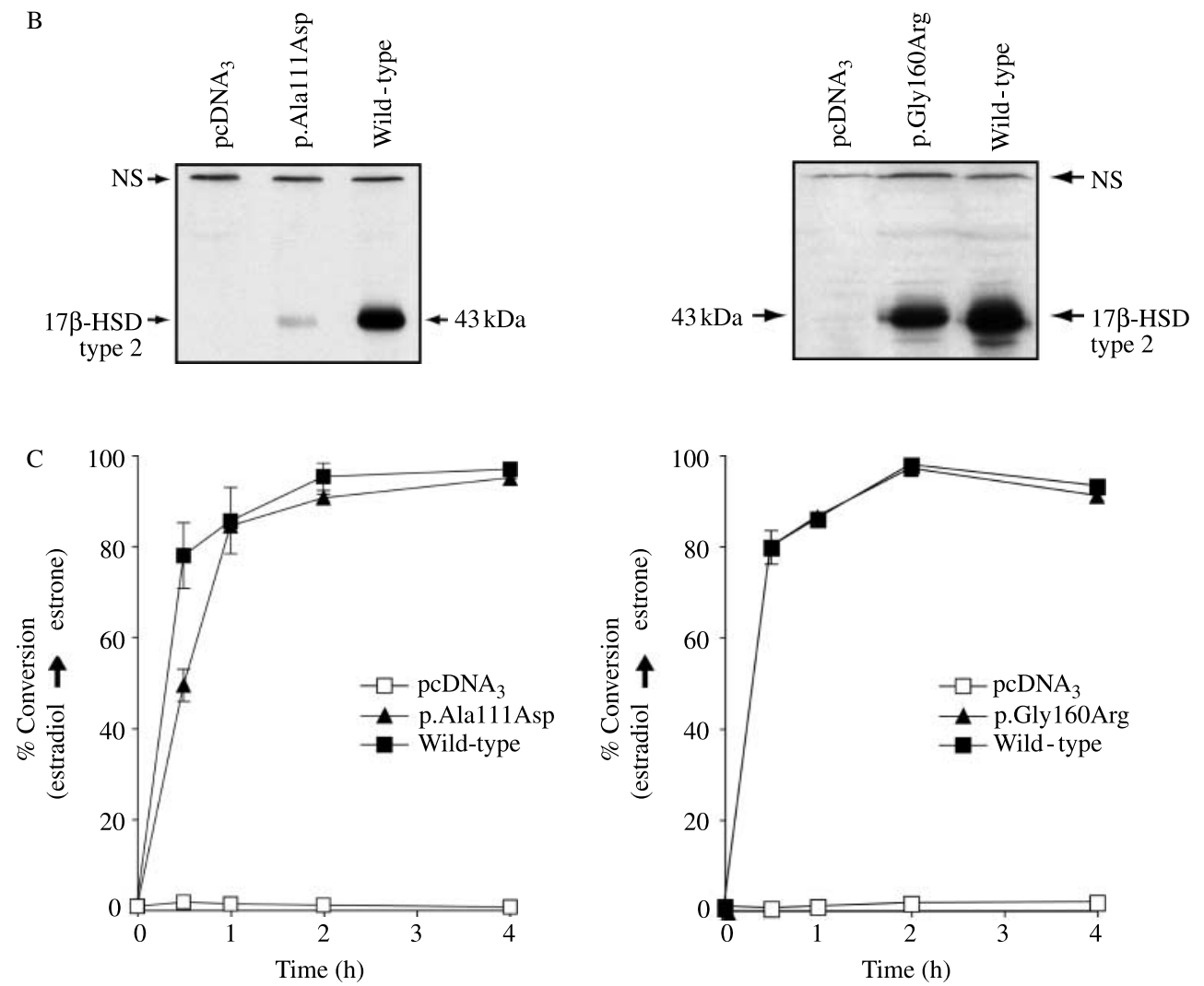

Figure 2 Comparison of expression levels and stability of mutant recombinant 17 $\beta-H S D$ type 2 proteins. (A) Representation of an in vitro transcription/translation (TNT) rabbit reticulocyte lysate assay showing that each pcDNA 3 construct is adequately translated into a $\left[{ }^{35} \mathrm{~S}\right.$-labeled-43 $\mathrm{kDa}$ protein, indicative of normal expression levels of mutant recombinant $17 \beta-H S D$ type 2 proteins. Translation was assessed by separation on a $12 \%$ SDS-PAGE gel. (B) Western blot analysis of homogenates purified from the corresponding HEK293 cells transiently transfected with the indicated expression vectors. A $43 \mathrm{kDa}$ band corresponding to $17 \beta$-hydroxysteroid dehydrogenase type 2 protein is detected in homogenate preparations from HEK293 transfected cells expressing wild-type and mutant recombinant protein p.Gly160Arg, while a weak signal is detected in cells transfected with the mutant recombinant protein p.Ala111Asp. No $43 \mathrm{kDa}$ protein is observed in mock $\mathrm{pCDNA}_{3}$ vector homogenates. The nonspecific band observed may be used as an internal control for loading. (C) Comparison of the time course of enzymatic conversion of $\left[{ }^{14} \mathrm{C}\right]$-estradiol into $\left[{ }^{14} \mathrm{C}\right]$-estrone in HEK293 cell homogenates transfected with the indicated expression vectors after normalization of the amount of immunoreactive mutant recombinant proteins to equivalent normal $17 \beta$-HSD type 2 protein expression for each cell homogenate preparation. The results are presented as the mean IS.E.M. $(n=3)$ and when the S.E.M. overlaps with the symbol used, only the symbol is illustrated. The cells were transfected with the pcDNA $A_{3}$ vector alone to show the absence of endogenous $17 \beta-H S D$ type 2 mRNA expression. 
at the HSD17B2 locus does not decrease significantly with distance. Seven haplotypes were estimated by PHASE from the six sequence variants found in the French Canadian population and haplotype frequencies were similar in cases and controls (data not shown). Our results, however, should be taken with caution since our study sample is of modest size and large sample sizes are required to obtain reliable results. Out of the seven haplotypes, only three exhibited a frequency of $\geq 5 \%$, representing $93 \cdot 1 \%$ of all observed haplotypes. Variants \#2 and \#5 can be used as tSNPs to predict these common haplotypes in future large-scale association studies.

\section{Discussion}

Since the discovery of susceptibility genes, such as $B R C A 1$ and BRCA2, that harbor many rare high-risk variants, failure to find strong evidence of new linkages has led some to argue that few genes harboring high-risk breast cancer susceptibility alleles remain to be identified, although there could still be individually rare variants in a number of genes that confer high risk. Homogenous family selection through close attention to tumor phenotype and/or from more homogenous populations, such as the French Canadian founder population, increases the likelihood of identifying genes harboring true highrisk variants. Based on the pivotal role of $H S D 17 B 2$ gene product in the estrogen metabolism pathway, it may be considered as a putative candidate gene that could possibly explain a fraction of the remaining familial breast cancer risk. However, no deleterious germline mutations leading to a premature termination of the protein were identified in the coding region. Therefore, $H S D 17 B 2$ is unlikely to play a role as a high penetrance gene in breast cancer predisposition.

On the other hand, it is likely that much of the unexplained familial risk is due to alleles of low to moderate penetrance. The genetic association study is a powerful tool for identifying such alleles. Although six sequence variants were identified in this study, among which two are novel missense variants, the possible involvement of variants or haplotypes observed in cases compared with those found in controls would need to be analyzed in a much larger sample set (Pharoah $\mathrm{et} \mathrm{al}$. 2002, Antoniou \& Easton 2006, Khoury et al. 2007). Indeed, until recently, most studies lacked the power to detect moderate relative risks at stringent levels of statistical significance, thus large sample sizes are needed to detect and confirm genetic variants that are associated with modest increases in risk. The ability to identify such genetic variants can be further improved by careful selection of both candidate gene and candidate polymorphism (Cox et al. 2007, Easton et al. 2007, Khoury et al. 2007). We thus sought to identify tagging SNPs that could be useful to other studies and populations. Our $\left|D^{\prime}\right|$ values of LD should be interpreted with caution because a small sample size tends to show higher levels of LD based on the mean of $\left|D^{\prime}\right|$ (Teare et al. 2002). On the other hand, variants \#2 (c.-133C $>$ T) and \#5 (c.803-57C $>$ G) are frequent variants and are in strong $\mathrm{LD}$, even if they are distantly spaced over $63 \mathrm{~kb}$. These findings suggest that $H S D 17 B 2$ gene is a region of strong LD with limited haplotype diversity and these sequence variants may be used as markers in case-control association studies using large cohorts to investigate their potential association with breast cancer risk (Pharoah et al. 2004).

Recently, after the completion of this study, Jansson et al. (2007) published their results from the HSD17B2 mutation screening among breast cancer cases from Sweden. No mutation was detected, but they did identify a previously unknown missense variant (p.Met226Val). They observed 5 and 15 heterozygotes for this variant among the 122 'hereditary' breast cancer cases and 457 controls respectively. This missense variant was not observed among the 50 affected individuals of the present study. In light of this very recent report, the presence of this variant was also ascertained in 69 French Canadian healthy controls and only one heterozygote was found. Further studies using larger sample sets will be needed to confirm that the p.Met226Val rare sequence variant is less frequent among the French Canadian population compared with the Swedish population.

In conclusion, no deleterious germline mutation potentially involved in breast cancer susceptibility has been identified in this French Canadian sample set. Indeed, data obtained from our functional assays taken together with the absence of co-segregation with breast cancer in family members do not support the association of p.Gly160Arg and p.Ala111Asp sequence variants with clustering of breast cancer in these families. Nevertheless, the possibility that a common sequence variant might have a differential effect in another ethnic group via gene-gene or gene-environment interactions, or that a predisposing sequence variant might be present exclusively in another population can not be excluded. The present study also demonstrates that p.Ala111Asp sequence variant seems to affect protein stability, a situation reminiscent

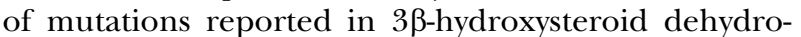
genase/ $\Delta 5-\Delta 4$ isomerase type 2 gene (Moisan et al. 1999, Simard et al. 2005). The in vivo functional implications of this observation remain to be determined, as are the possible functional consequences of the other observed sequence variants. Considering the importance of HSD17B2 gene, the complexities of the susceptibility to breast cancer, and 
the limitations of our study, additional population studies using larger cohorts are warranted to define the possible association of $H S D 17 B 2$ gene sequence variants with breast cancer susceptibility.

\section{Acknowledgements}

The authors are indebted to the participants and their families for their generosity and providing DNA samples. We thank Dr Damian Labuda from Centre de cancérologie Charles Bruneau, Ste-Justine Hospital, for control DNA samples. We would like to thank Martine Tranchant for his skillful technical assistance as well as Tina Babineau, Nathalie Bolduc, Claire Brousseau, MarieAndrée Lajoie, Pascale Léger, Hélène Malouin, Andrée McMillan, and Josée Rhéaume for their genetic counseling and clinical data management at the Cancer Genomics Laboratory. We also thank Geneviève Ouellette for establishment of EBV-transformed B-lymphoblastoid cell lines and RNA and genomic DNA extractions. Expression vector containing the cDNA fragment encoding the human $17 \beta$-HSD type 2 and the antibody against the $17 \beta-H S D$ type 2 protein were kindly provided by Dr Van Luu-The from the CHUL Research Center. We thank Claudia Moreau at the Centre de Recherche de l'Hôpital Ste-Justine for help with control DNA samples.

\section{Disclosure}

This work was supported by the Canadian Institutes of Health Research (CIHR) through the INHERIT BRCAs research program, the Fonds de la Recherche en Santé du Québec (FRSQ) / Réseau de Médecine Génétique Appliquée (RMGA), the Canadian Breast Cancer Research Alliance and the CURE Foundation. J S is a Chairholder of the Canada Research Chair in Oncogenetics. F D is a recipient of a chercheur-boursier from the Fonds de la Recherche en Santé du Québec (FRSQ) and a Research Career Award in the Health Sciences from CIHR/Rx\&DH Research Foundation. The authors declare that there is no conflict of interest that would prejudice the impartiality of this scientific work.

\section{References}

Alex Dong Li's SpliceSiteFinder. http://www.genet.sickkids.on.ca/ali/ splicesitefinder.html.

Antoniou AC \& Easton DF 2006 Models of genetic susceptibility to breast cancer. Oncogene 25 5898-5905.

Antoniou AC, Pharoah PD, McMullan G, Day NE, Ponder BA \& Easton D 2001 Evidence for further breast cancer susceptibility genes in addition to BRCA1 and BRCA2 in a population-based study. Genetic Epidemiology 21 1-18.
Antoniou AC, Durocher F, Smith P, Simard J \& Easton DF 2006 BRCA1 and BRCA2 mutation predictions using the BOADICEA and BRCAPRO models and penetrance estimation in high-risk FrenchCanadian families. Breast Cancer Research 8 R3.

Cargill M \& Daley GQ 2000 Mining for SNPs: putting the common variants-common disease hypothesis to the test. Pharmacogenomics $127-37$.

Casey ML, MacDonald PC \& Andersson S 1994 17ß-Hydroxysteroid dehydrogenase type 2: chromosomal assignment and progestin regulation of gene expression in human endometrium. Journal of Clinical Investigation 94 2135-2141.

Cleton-Jansen AM, Callen DF, Seshadri R, Goldup S, McCallum B, Crawford J, Powell JA, Settasatian C, van Beerendonk H, Moerland EW et al. 2001 Loss of heterozygosity mapping at chromosome arm $16 q$ in 712 breast tumors reveals factors that influence delineation of candidate regions. Cancer Research 61 1171-1177.

Cox A, Dunning AM, Garcia-Closas M, Balasubramanian S, Reed MW, Pooley KA, Scollen S, Baynes C, Ponder BA, Chanock S et al. 2007 A common coding variant in CASP8 is associated with breast cancer risk. Nature Genetics 39 352-358.

Devlin B \& Risch N 1995 A comparison of linkage disequilibrium measures for fine-scale mapping. Genomics 29 311-322.

Ding K, Zhou K, He F \& Shen Y 2003 LDA - a java-based linkage disequilibrium analyzer. Bioinformatics 19 2147-2148.

Doisneau-Sixou SF, Sergio CM, Carroll JS, Hui R, Musgrove EA \& Sutherland RL 2003 Estrogen and antiestrogen regulation of cell cycle progression in breast cancer cells. Endocrine-Related Cancer 10 179-186.

Durocher F, Labrie Y, Soucy P, Sinilnikova O, Labuda D, Bessette P, Chiquette J, Laframboise R, Lepine J, Lesperance B et al. 2006 Mutation analysis and characterization of ATR sequence variants in breast cancer cases from high-risk French Canadian breast/ovarian cancer families. BMC Cancer 6 230-250.

Easton DF, Pooley KA, Dunning AM, Pharoah PD, Thompson D, Ballinger DG, Struewing JP, Morrison J, Field H, Luben R et al. 2007 Genome-wide association study identifies novel breast cancer susceptibility loci. Nature 447 1087-1093.

Fearnhead NS, Wilding JL, Winney B, Tonks S, Bartlett S, Bicknell DC, Tomlinson IP, Mortensen NJ \& Bodmer WF 2004 Multiple rare variants in different genes account for multifactorial inherited susceptibility to colorectal adenomas. PNAS 101 15992-15997.

Feigelson HS, McKean-Cowdin R, Coetzee GA, Stram DO, Kolonel LN \& Henderson BE 2001 Building a multigenic model of breast cancer susceptibility: CYP17 and HSD17B1 are two important candidates. Cancer Research 61 785-789.

Feigelson HS, Cox DG, Cann HM, Wacholder S, Kaaks R, Henderson BE, Albanes D, Altshuler D, Berglund G, Berrino F et al. 2006 Haplotype analysis of the HSD17B1 gene and risk of breast cancer: a comprehensive approach to multicenter analyses of prospective cohort studies. Cancer Research 66 2468-2475.

Gunnarsson C, Olsson BM \& Stal O 2001 Abnormal expression of 17beta-hydroxysteroid dehydrogenases in breast cancer predicts late recurrence. Cancer Research 61 8448-8451.

Gunnarsson C, Hellqvist E \& Stal O 2005 17ß-Hydroxysteroid dehydrogenases involved in local oestrogen synthesis have prognostic significance in breast cancer. British Journal of Cancer 92 547-552.

Hill WG \& Robertson A 1968 Linkage disequilibrium in finite populations. Theoretical and Applied Genetics 38 226-231.

Jansson A, Carlsson J, Olsson A, Storm P, Margolin S, Gunnarsson C, Stenmark-Askmalm M, Lindblom A, Persson B \& Stal O 2007 A new polymorphism in the coding region of exon four in HSD17B2 in relation to risk of sporadic and hereditary breast cancer. Breast Cancer Research and Treatment 10 57-64.

Khoury MJ, Little J, Gwinn M \& Ioannidis JP 2007 On the synthesis and interpretation of consistent but weak gene-disease associations in the era of genome-wide association studies. International Journal of Epidemiology 36 439-445. 
Labrie Y, Durocher F, Lachance Y, Turgeon C, Simard J, Labrie C \& Labrie F 1995 The human type II 17beta-hydroxysteroid dehydrogenase gene encodes two alternatively spliced mRNA species. DNA and Cell Biology 14 849-861.

Labrie F, Luu-The V, Labrie C, Belanger A, Simard J, Lin SX \& Pelletier G 2003 Endocrine and intracrine sources of androgens in women: inhibition of breast cancer and other roles of androgens and their precursor dehydroepiandrosterone. Endocrine Reviews 24 152-182.

Lewontin RC 1964 The interaction of selection and linkage. I. General considerations; heterotic models. Genetics 49 49-67.

Lichtenstein P, Holm NV, Verkasalo PK, Iliadou A, KaprioJ, Koskenvuo M, Pukkala E, Skytthe A \& Hemminki K 2000 Environmental and heritable factors in the causation of cancer - analyses of cohorts of twins from Sweden, Denmark, and Finland. New England Journal of Medicine 343 78-85.

Lohmueller KE, Pearce CL, Pike M, Lander ES \& Hirschhorn JN 2003 Meta-analysis of genetic association studies supports a contribution of common variants to susceptibility to common disease. Nature Genetics 33 177-182.

Mannermaa A, Peltoketo H, Winquist R, Ponder BA, Kiviniemi H, Easton DF, Poutanen M, Isomaa V \& Vihko R 1994 Human familial and sporadic breast cancer: analysis of the coding regions of the 17 beta-hydroxysteroid dehydrogenase 2 gene (EDH17B2) using a single-strand conformation polymorphism assay. Human Genetics 93 319-324.

Miettinen M, Mustonen M, Poutanen M, Isomaa V, Wickman M, Soderqvist G, Vihko R \& Vihko P 1999 17Beta-hydroxysteroid dehydrogenases in normal human mammary epithelial cells and breast tissue. Breast Cancer Research and Treatment 57 175-182.

Mindnich R, Moller G \& Adamski J 2004 The role of $17 \beta$-hydroxysteroid dehydrogenases. Molecular and Cellular Endocrinology 218 7-20.

Moisan AM, Ricketts ML, Tardy V, Desrochers M, Mebarki F, Chaussain JL, Cabrol S, Raux-Demay MC, Forest MG, Sippell WG et al. 1999 New insight into the molecular basis of $3 \beta$ hydroxysteroid dehydrogenase deficiency: identification of eight mutations in the HSD3B2 gene eleven patients from seven new families and comparison of the functional properties of twentyfive mutant enzymes. Journal of Clinical Endocrinology and Metabolism 84 4410-4425.

Moisan AM, Fortin J, Dumont M, Samson C, Bessette P, Chiquette J, Laframboise R, Lepine J, Lesperance B, Pichette R et al. 2006 No evidence of BRCA1/2 genomic rearrangements in high-risk FrenchCanadian breast/ovarian cancer families. Genetic Testing 10 104-115.

Ng PC \& Henikoff S 2001 Predicting deleterious amino acid substitutions. Genome Research 11 863-874.

Oduwole OO, Li Y, Isomaa VV, Mantyniemi A, Pulkka AE, Soini Y \& Vihko PT 2004 17ß-hydroxysteroid dehydrogenase type 1 is an independent prognostic marker in breast cancer. Cancer Research $\mathbf{6 4}$ 7604-7609.

Oppermann UC, Filling C \& Jornvall H 2001 Forms and functions of human SDR enzymes. Chemico-Biological Interactions 130-132 $699-705$.

Peto J \& Mack TM 2000 High constant incidence in twins and other relatives of women with breast cancer. Nature Genetics 26 411-414.

Pharoah PD, Antoniou A, Bobrow M, Zimmern RL, Easton DF \& Ponder BA 2002 Polygenic susceptibility to breast cancer and implications for prevention. Nature Genetics 31 33-36.

Pharoah PD, Dunning AM, Ponder BA \& Easton DF 2004 Association studies for finding cancer-susceptibility genetic variants. Nature Reviews. Cancer 4 850-860.

Plourde M, Samson C, Durocher F, Sinilinikova O, INHERIT \& Simard J 2007 Characterization of HSD17B1 sequence variants in breast cancer cases from French Canadian families with high risk of breast and ovarian cancer. Journal of Steroid Biochemistry and Molecular Biology (in press) doi: 10.1016/j.jsbmb.2007.10.004.

Pritchard JK 2001 Are rare variants responsible for susceptibility to complex diseases? American Journal of Human Genetics 69 124-137.
Pritchard JK \& Cox NJ 2002 The allelic architecture of human disease genes: common disease-common variant or not? Human Molecular Genetics 11 2417-2423.

Rahman N, Seal S, Thompson D, Kelly P, Renwick A, Elliott A, Reid S, Spanova K, Barfoot R, Chagtai T et al. 2007 PALB2, which encodes a BRCA2-interacting protein, is a breast cancer susceptibility gene. Nature Genetics 39 165-167.

Ramensky V, Bork P \& Sunyaev S 2002 Human non-synonymous SNPs: server and survey. Nucleic Acids Research 30 3894-3900.

Reich DE \& Lander ES 2001 On the allelic spectrum of human disease. Trends in Genetics 17 502-510.

Renwick A, Thompson D, Seal S, Kelly P, Chagtai T, Ahmed M, North B, Jayatilake H, Barfoot R, Spanova K et al. 2006 ATM mutations that cause ataxia-telangiectasia are breast cancer susceptibility alleles. Nature Genetics 38 873-875.

Sato M, Mori Y, Sakurada A, Fukushige S, Ishikawa Y, Tsuchiya E, Saito Y, Nukiwa T, Fujimura S \& Horii A 1998 Identification of a 910-kb region of common allelic loss in chromosome bands 16q24.1-q24.2 in human lung cancer. Genes, Chromosomes and Cancer 22 1-8.

Seal S, Thompson D, Renwick A, Elliott A, Kelly P, Barfoot R, Chagtai T, Jayatilake H, Ahmed M, Spanova K et al. 2006 Truncating mutations in the Fanconi anemia J gene BRIP1 are low-penetrance breast cancer susceptibility alleles. Nature Genetics 38 1239-1241.

Setiawan VW, Hankinson SE, Colditz GA, Hunter DJ \& De Vivo I 2004 HSD17B1 gene polymorphisms and risk of endometrial and breast cancer. Cancer Epidemiology, Biomarkers and Prevention 13 213-219.

Shapiro MB \& Senapathy P 1987 RNA splice junctions of different classes of eukaryotes: sequence statistics and functional implications in gene expression. Nucleic Acids Research 15 7155-7174.

Simard J, Ricketts ML, Gingras S, Soucy P, Feltus FA \& Melner MH

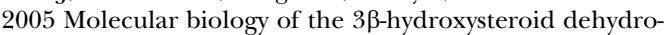
genase / $\Delta 5-\Delta 4$ isomerase gene family. Endocrine Reviews 26 525-582.

Simard J, Dumont M, Moisan AM, Gaborieau V, Malouin H, Durocher F, Chiquette J, Plante M, Avard D, Bessette P et al. 2007 Evaluation of BRCA1 and BRCA2 mutation prevalence, risk prediction models and a multistep testing approach in French-Canadian families with high risk of breast and ovarian cancer. Journal of Medical Genetics $\mathbf{4 4}$ 107-121.

Splice Site Prediction by Neural Network. Berkeley Drosophilia Genome Project: http://www.fruitfly.org/seq_tools/splice.html.

Stephens M, Smith NJ \& Donnelly P 2001 A new statistical method for haplotype reconstruction from population data. American Journal of Human Genetics 68 978-989.

Suzuki H, Komiya A, Emi M, Kuramochi H, Shiraishi T, Yatani R \& Shimazaki J 1996 Three distinct commonly deleted regions of chromosome arm $16 \mathrm{q}$ in human primary and metastatic prostate cancers. Genes, Chromosomes and Cancer 17 225-233.

Teare MD, Dunning AM, Durocher F, Rennart G \& Easton DF 2002 Sampling distribution of summary linkage disequilibrium measures. Annals of Human Genetics 66 223-233.

Thompson D \& Easton D 2004 The genetic epidemiology of breast cancer genes. Journal of Mammary Gland Biology and Neoplasia 9 221-236.

Wu L, Einstein M, Geissler WM, Chan HK, Elliston KO \& Andersson S 1993 Expression cloning and characterization of human $17 \beta-$ hydroxysteroid dehydrogenase type 2 , a microsomal enzyme possessing 20 alpha-hydroxysteroid dehydrogenase activity. Journal of Biological Chemistry 268 12964-12969.

Wu AH, Seow A, Arakawa K, Van Den Berg D, Lee HP \& Yu MC 2003 HSD17B1 and CYP17 polymorphisms and breast cancer risk among Chinese women in Singapore. International Journal of Cancer 104 $450-457$.

Received in final form 28 November 2007

Accepted 21 January 2008

Made available online as an Accepted Preprint

21 January 2008 


\section{Appendix}

Other members of INHERIT BRCAs involved in clinical aspects of the program.

Paul Bessette: Department of Obstetrics and Gynecology, Centre Hospitalier Universitaire de Sherbrooke, Fleurimont, QC, Canada

Jocelyne Chiquette: Clinique des maladies du sein Deschênes-Fabia, Hôpital du Saint-Sacrement, Québec, QC, Canada

Rachel Laframboise: Medical Genetics Division, Centre Hospitalier Universitaire de Québec,
CHUL, Laval University, Québec, QC, Canada Jean Lépine: Haemato-Oncology Service, Centre Hospitalier Régional de Rimouski, Rimouski, QC, Canada

Bernard Lespérance, Roxanne Pichette: Department of Haemato-Oncology, Hôpital du Sacré-Coeur de Montréal, Montréal, QC, Canada

Marie Plante: Gynecology Oncology Division, Hôtel-Dieu de Québec, Centre Hospitalier Universitaire de Québec, Laval University, Québec, QC, Canada 